\title{
Does TRX training induce similar effects to crossfit? Study on the variation of body fat mass, endurance and explosive force
}

\author{
Angela Notarnicola ${ }^{1,2}$ \\ Giuseppe Salatino ${ }^{2}$ \\ Paolo Napoletano² \\ Antonio Monno ${ }^{2}$ \\ Biagio Moretti ${ }^{1,2}$ \\ Silvio Tafuri ${ }^{1,3}$
}

${ }^{1}$ Course of Motor and Sports Sciences, Department of Medical Sciences of Basis, Neurosciences and Organs of Sense, Faculty of Medicine and Surgery, University of Study of Bari, Bari, Italy

2 Orthopedics Section, Department of Medical Sciences of Basis, Neurosciences and Organs of Sense, Faculty of Medicine and Surgery, University of Study of Bari, General Hospital, Bari, Italy

${ }^{3}$ Department of Biomedical Sciences and Human

Oncology, Faculty of Medicine and Surgery, University of Study of Bari, General Hospital, Bari, Italy

Corresponding author:

Angela Notarnicola

UOC Ortopedia e Traumatologia

70124 Piazza Giulio Cesare 11

Bari, Italy

Tel.: 0805592938

E-mail: angelanotarnicola@yahoo.it

\section{Summary}

Introduction: Though TRX suspension training and CrossFit are similar methods of functional training, until now the differences in the effects on body composition and motor skills are not known.

Methods: A sample of CrossFitters and TRXers was enrolled and evaluated at the beginning of the study and after 6 months. body mass index (BMI), waist-to-height-ratio (WHtR), body fat percentage, Sargent test and Balke test were used for the evaluation of the two groups.

Results: Fat percentage, WHtR showed a significant improvement over time, without statistical significant differences between the two groups. No statistical differences were found between groups for aerobic capacity, even if both present an improvement for all parameters during time $(p<0.05)$. The study revels also a greater increase for VO2max values in CrossFit group instead of TRX group, confirmed by the fact that changes in the values are related to the treatment group $(p=0.01)$. The average mean score for the Sargent test revealed how the modification of the values are explained for time $(p=0.0002)$ and for groups $(p=0.009)$.

Conclusion: Our study shows that both functional training methods lead to improvements both from an anthropometric point of view and from an aerobic resistance point of view. Additionally, CrossFit athletes method reveals a lower limb explosiveness and VO2max high values than the TRX group. Level of evidence: IIIb.

KEY WORDS: functional training, endurance, fat mass, aerobic resistance.

\section{Introduction}

Functional training (FT) represents a new way of understanding physical activity for years ${ }^{1}$. The word "functional" refers to the performance of an action, work or activity ${ }^{2}$. In particular, functional exercise training programs should be designed to imitate the activities and movement patterns that occur in an athlete or person characteristic activity (activity of daily living, ADL, job, sports, etc.) with the purpose to make training adaptations more specific. Functional fitness has been defined as emphasizing multiple muscle and joint activities, combining upper and lower body movements, utilizing and optimizing the entire body in each movement ${ }^{3}$. This idea represents the promoter of the notion that FT should be designed to improve movement-based exercises throughout flexibility, core stability, strength training, balance activity, etc., thus avoiding focusing exclusively on specific muscular adaptations, as in traditional strength training.

FT attempts to train muscles in coordinated, multiplanar movement patterns and incorporates multiple joints, dynamic tasks, and consistent alterations in the base of support for the purpose of improving function ${ }^{4,5}$. Trainers and coaches affirm that FT as purposeful training stating that "function is, essentially, purpose" $4,6,7$. Therefore, functional training can be any type of training that is performed with purpose to enhance a certain movement or activity. So, FT could be defined as a generic workout where not only one feature is required. Being functional means being strong, reactive, agile, fast, elastic, coordinated, thanks to the 
fact that new motor patterns are acquired through multiple and increasingly difficult motor experiences during the training sessions. Functional training modifies the body composition reducing the fat and improves coordination, strength, agility and aerobic capacity in studied populations, as post-menopausal women ${ }^{8}$, adult cancer survivors ${ }^{9}$ and military ${ }^{10}$.

In the last decades, the two-main effective approach to functional training are represented by CrossFit and $T R X^{\circledR}$ suspension training. CrossFit is physical strength and conditioning program and constantly varied functional movements performed at high intensity, such as weightlifting, gymnastics and metabolic conditioning ${ }^{11}$. Training is planned as daily sessions named "workouts of the day" or WOD. Exercises change continuously. All CrossFit WODs are based on functional movements, and these movements reflect the best aspects of gymnastics, weightlifting, running, rowing and more. These are the core movements of life. The community that spontaneously arises when people do these workouts together is a key component of why CrossFit is so effective, and it gave birth to a global network of CrossFit affiliates that number over 13,000, about half of them are in United States ${ }^{12}$. In Europe, the number of affiliates doubled every year since $2009^{13}$. Early studies are starting to test the effects of CF on metabolism and motor skills ${ }^{10-13}$. In a study included Korean college students, 14 weeks of CF exercises are responsible of reduction of weight and body mass index (BMI) and improvement of muscle mass, without modification of blood profiles ${ }^{14}$. The CF program was efficacious in reducing waist circumference and $\mathrm{BMI}$ in adolescents ${ }^{15}$.

With regard to the TRX, the first version of this training program was created in $1997^{16}$. In 2005 TRX launched the first suspension training and 5 years after launch, TRX had more than one million users in over 60 countries. The main concept of the TRX is to develop strength, to burn fat and improve heart health through different and numerous exercises ${ }^{16}$. Nowadays, there are 250,000 TRX professionals and 40,000 Olympians and MMA (mixed martial arts) fighters using TRX as training tool for improving their performance. Actually the scientific insights on TRX have focused only on analyzing the effects of this training on muscle recruitment ${ }^{16}$. TRX® suspension training induces strengthening of the core muscles and guarantees stability and induces specific adaptations in athletes ${ }^{16}$. Till now no studies verified the effects of this type of training program on changes in body composition and resistance.

Considering the analogy between CF and TRX, it is rational to compare these two methods, in particular for the effects on body composition, resistance and explosive-elastic force of the lower limb that we know are better after CF. The hypothesis to verify is if the two training methods induce similar effects, not having any data on the TRX for now.

\section{Methods}

\section{Population and study design}

We conducted a perspective cohort study, with the sequential recruitment of CrossFitters (CF) and TRXers (TRX) belonging to a gym of our city. Informed consent was requested to each participant. Enrolled athletes did not receive money to participate to the study.

The research was conducted ethically according to international standards and as required by the journal ${ }^{17}$. The inclusion criteria were: age from 18 to 40 years and the practice of CrossFit or TRX for at least 6 months. CrossFit enrolled athletes have to attend training not less than 3 times a week while TRX athletes not less than 2 times a week. The exclusion criteria: obesity $\left(\mathrm{BMI}>30 \mathrm{~kg} / \mathrm{m}^{2}\right)$, recent traumas (less than two months) at the moment of measurements and performing other sports than CrossFit and TRX.

The two training models were designed and executed respectively following the recommendations of Crossfit.inc ${ }^{18}$ in CF group and the recommendation of TRX academy $^{19}$ for TRX group. All athletes were properly informed of the nature of the study and they signed an informed consent document according to the Declaration of Helsinki and the protocol was fully approved by the Clinical Research Ethics Committee.

\section{Measures}

Athletes were evaluated at the start of the study (TO) and after 6 months (T1): BMI, waist-to-height-ratio (WHtR), body fat percentage (FAT\%), Sargent test and Balke test were used in the evaluation. For each of the subjects enrolled, a data collection form was compiled including personal data, anthropometric measurements and the results of the tests. We encountered no refusal and the measurements were performed by the same person (PN) to minimize inter-observer variability. The used morphological measurements were: body mass (weight) $(\mathrm{kg})$ was measured twice (Seca ${ }^{\circledR} 861$ scales) with the athlete barefoot and in light clothing; height $(\mathrm{cm})$ was measured twice, using a wall stadiometer (Kawe ${ }^{\circledR} 222$ ), with the athlete barefoot and upright and with the sagittal midline touching the back board. Body mass index $\left(\mathrm{kg} / \mathrm{m}^{2}\right)$ was calculated as weight in kilograms divided by the square of the height in meters. BMI score was evaluated according to the International classification of underweight, normal range and obese ${ }^{20}$.

Waist circumference (WC) (cm) was measured at 2 $\mathrm{cm}$ below the navel at the end of a normal expiration. Waist diameter $(\mathrm{cm})$ was measured at the pelvis at the widest points, at the end of a normal expiration. The circumference was measured two times using a flexible tape (Amtech P1800). WHtR was calculated by dividing waist circumference in centimeters by height in centimeters ${ }^{21}$. WHtR was used to measure the distribution of body fat. Higher values of $\mathrm{WHtR}$ indicate higher risk of obesity-related cardiovascular diseases $^{22}$. 


\section{Plicometry}

Non dominant skinfold thicknesses were measured using a commercial caliper (GIMA Professional Mechanical Plicometer) at the following sites: triceps, suprailiac and thigh skinfolds for women; chest, abdomen and thigh skinfolds for men. Skinfold thicknesses were assessed three times and the mean of three readings was taken. These data were used to calculate the percentage of fat mass on the total body mass. Results of plicometry were interpreted according to Jackson and Pollock equation ${ }^{23}$.

\section{Functional tests}

All the athletes performed functional tests: Balke Treadmill Test and Sargent Test.

Balke Treadmill Test ${ }^{24}$ was developed as clinician test to determine peak of VO2 in cardiac patients, though it can also be used to estimate cardiovascular fitness in athletes. The athletes walk on a treadmill to exhaustion, at a constant walking speed while the gradient (slope) is increased every one or two minutes. The assistant starts the stopwatch at the beginning of the test and stops it when the subject is unable to continue. For men, the treadmill speed was set at $5.3 \mathrm{~km} / \mathrm{h}$, with the gradient starting at $0 \%$. After 1 minute, it was raised to $2 \%$ with constant velocity. After 2 minutes ( $3^{\text {rd }}$ minute) it is raised to another $1 \%$. Starting from this time on, every minute the gradient was increased by one percentage point. For women the treadmill speed was set at $4.8 \mathrm{~km} / \mathrm{h}$, with the gradient starting at $0 \%$. After 3 minutes, it was raised to $3 \%$ with constant velocity. Starting from this time, every three minute the gradient was increased by 2.5 percentage point. In both cases, the test ended when the subjects were not able to bear additional slope increments. At the end of the test, the following values were registered: Maximum Heart Rate (MHR), Absolute VO2max (VO2max), Relative VO2max, Metabolic Equivalent of Task (MET). During the tests, was took into considerations for estimating the pre and post workout evaluation with the trainer, regarding the estimate of the relative VO2max. The results were compared with normative data for VO2max ${ }^{25}$.

The Sargent test ${ }^{26}$ was used to measure the explosive strength of lower limbs. In order to carry out the test, a wall, a chalk and a measuring tape were needed. According to the protocol, the athletes stands side on to a wall and reaches up with the hand closest to the wall. Keeping the feet flat on the ground, the point of the fingertips is marked. The athlete stands out from the wall and jump vertically to the highest possible point with a squat loading expressing his maximum explosive capacity. They marked with the chalk on the wall their maximum height reached. The test has to be performed three times and measures were evaluated by two strength and conditioning experts. In case of differences between the two evaluations, the test was repeated. The difference in distance between the standing reach height and the jump height is the score. The mean of the three attempts is recorded. The data were compared with Sargent test reference values ${ }^{27}$.

\section{Statistical analysis}

Data from each subject enrolled were reported in a form, that contains: demographic data, anthropometric measures and functional tests performed at T0 and T1. Completed forms were inputted in a database created by Google Drive and STATA MP14 was used for all statistical analyses. The mean and standard deviation was computed for all the quantitative variables and the normality of the distribution was measured. Qualitative variables were computed as percentage with confidence interval set at $95 \%$. The following tests were used for the data analysis. Student's $t$-test was performed for comparisons of means between the two groups (CF, TRX). To compare means at different time for each group, a paired t-test was run. An analysis of variance (ANOVA) for repeated measures was performed to assess whether the parameters computed were significantly different between groups. Chi-square was used to compare the proportions between groups. For all test the significance level was set at $p<0.05$.

\section{Results}

Sixteen CrossFitters (CF) and sixteen TRXers (TRX) were enrolled. Both groups were matched for gender (8 males and 8 females in each group). Average age did not differ between the two groups $(23.5 \pm 4.7 \mathrm{CF}$ 30.3 \pm 8.7 TRX; $p>0.05)$. Demographic and anthropometric characteristics of the two groups are presented in Table I. The BMI values do not seem to differ per group ( $\mathrm{F}=0.01 ; p=0.93)$ or per time $(\mathrm{F}=1.74 ; p=19.7)$ or there is no group-time interaction ( $F=0.02 ; p=0.88)$. However, WHtR and FAT\% did not show statistically significant differences between groups $(p>=0.05)$, but their values change in relations to time [WHTR $(F=7.62$; $p=0.01)$; FAT\% $(\mathrm{F}=17.7 ; p=0.0002)]$.

\section{Functional tests}

The Balke test results are shown in Table II. Results obtained by the Balke test show that none of the parameters reveal group-time interaction. At the beginning of the study, only the MHR average value was higher in the CrossFit group compared to the TRX group $(\mathrm{t}=2.7 ; p=0.01)$. Concerning VO2Max, relativeVO2Max and MET values were similar between groups. Additionally, data obtained at the end of the study, shows also that only variations of the MHR values are dependent on the treatment group $(F=3.14$; $p=0.009$ ). No effects are observed on the membership group for all the other parameters. However, relative VO2max and METs values are changed during time [rVO2max $(\mathrm{F}=4.9 ; p=0.03) ; \operatorname{METs}(\mathrm{F}=4.7$; $p=0.04)$ ] compared to MHR and absolute VO2max.

The two groups were not homogeneous for the Sargent test results. The variations of this value are re- 
Table I. Demographic and anthropometric characteristics of enrolled subjects, per group. The mean value and standard deviation of variables analysed for each of the two groups and at the two times. T0: at the start of the study; T1: after 6 months. BMI: Body Mass index; WHtR: Waist-to-height ratio.

\begin{tabular}{lllll}
\hline & CrossFit & \multicolumn{3}{l}{ TRX } \\
\hline Variable & T0 & T1 & T0 & T1 \\
\hline BMI $\left(\mathrm{kg} / \mathrm{m}^{2}\right)$ & $22.9 \pm 2.1$ & $22.8 \pm 2.1$ & $22.8 \pm 2.7$ & $22.7 \pm 2.5$ \\
Weight $(\mathrm{kg})$ & $66.0 \pm 12.9$ & $66.0 \pm 12.9$ & $67.4 \pm 13.1$ & $67.4 \pm 13.1$ \\
WHtR (ratio) & $0.45 \pm 0.02$ & $0.44 \pm 0.02$ & $0.45 \pm 0.03$ & $0.44 \pm 0.02$ \\
Fat mass (\%) & $15.2 \pm 6.8$ & $13.3 \pm 5.6$ & $15.2 \pm 5.3$ & $14.2 \pm 5.4$ \\
\hline
\end{tabular}

Table II. Functional tests of enrolled subjects, per group. The mean value and standard deviation of variables analysed for each of the two groups and at the two times. T0: at the start of the study; T1: after 6 months. MET: Metabolic Equivalent of Task.

\begin{tabular}{lllll}
\hline & \multicolumn{3}{l}{ CrossFit } & \multicolumn{3}{l}{ TRX } \\
\hline Variable & T0 & T1 & T0 & T1 \\
\hline $\begin{array}{lllll}\text { Balke test (maximum } \\
\text { heart rate MHR) }\end{array}$ & $196.7 \pm 4.9$ & $196.6 \pm 4.9$ & $190 \pm 8.7$ & $189.9 \pm 8.6$ \\
Relative Vo2 max & $41.4 \pm 6.6$ & $44.3 \pm 6.0$ & $39.9 \pm 5.5$ & $41.7 \pm 5.0$ \\
Absolute Vo2 max & $2.8 \pm 0.8$ & $3.0 \pm 0.9$ & $2.7 \pm 0.8$ & $2.8 \pm 0.6$ \\
METs & $11.9 \pm 1.9$ & $12.6 \pm 1.6$ & $11.3 \pm 1.6$ & $11.9 \pm 1.4$ \\
Sargent Test $(\mathrm{cm})$ & $41.0 \pm 12.1$ & $43.3 \pm 12.8$ & $30.9 \pm 9.5$ & $32.1 \pm 9.0$ \\
\hline
\end{tabular}

lated both to time $(F=18.1, p=0.0002)$ and to the group ( $\mathrm{F}=7.8 ; p=0.009)$, while there is no group-time interaction $(\mathrm{F}=2.01 ; p=0.17)$.

\section{Discussion}

The aim of this research was to evaluate possible modification of anthropometric parameters and endurance and explosive force in a sample of non-professional CF and TRX athletes. In CF and TRX training there are a combination of resistance exercises with the high volumes of repetitive weightlifting motion that could explain the improving of both aerobic fitness and anaerobic power ${ }^{28-30}$.

In detail, weight and BMI do not present any significant variation in the two groups examined and over time. Based on the results of our observation, both groups show an improvement of the functional parameters investigated, without statistically significant short-term differences between the two groups. For parameters such as fat percentage, $\mathrm{WHtR}$, relative VO2max, VO2max, METs and Sargent test, there is a significant improvement over time. The most remarkable result is that the Sargent test and the value of VO2max shows an higher increase of values in the CrossFit group. As far as we know the results emphasizes the model of the CrossFit training and its methodology which includes plyometric exercises or jump box and all the exercises used for stimulating the lower limb explosive strength such as snatch, clean \& jerk, wall ball shots. In fact, as reported in literature, the clean and jerk and snatch lifts have the potential to produce some of the average human power outputs ${ }^{31,32}$. Furthermore, Manocchia et al. suggested how kettlebells training may be an effective alternative tool to improve performance in weightlifting and powerlifting ${ }^{33}$.

The heart rate in the CrossFit group is also high compared to the TRX group. As proposed by Pollock et al., the evidence we found points to a significant increase in the relative $\mathrm{VO} 2 \mathrm{max}$ and decrease in percent body fat observed in their study ${ }^{34}$. Through a regression analysis they also revealed that the absolute VO2max and body fat percentage were significant predictors of the change in the relative VO2max in men and also in women ${ }^{34}$.

Our work has led us to conclude that both functional training methods are lead to an improvement of the anthropometric measures and endurance strength. The results suggest that for CrossFit, in addition to improving the above listed qualities, there is an implementation of the explosive strength more than the TRX group. Pollock et al. ${ }^{34}$ demonstrated that exercises variables as frequency, intensity, and duration must be manipulated in order to create an 
overload with which the body is unfamiliar and to achieve a subsequent training effect. Probably, the efficacy of CrossFit instead of TRX is due to its high levels of training intensity, directly linked to the short time of the results. Generally, explosive exercises or speed strength exercises result in the production of high power outputs ${ }^{35}$. Thus, the use of explosive lifts such as the CrossFit exercises may partially explain the differences in power output capabilities of different strength power athletes. In addition, the differences in some anthropometric measurements between CrossFit and TRX may be related to the use of these explosive exercises which stimulate the improvement of output-generating capabilities, produce movement patterns, velocity characteristics and power outputs similar to those needed in sports performances.

This is one of the first scientific studies that compared two important and emerging functional training methods; one of the most important strength is related with the setting of the study. Subjects performed the tests in one gym centre, using the same tools and with the supervision of the same group of trainers. The study has some limitations as the low number of subjects enrolled and the short time of follow-up. Besides, the study did not include food diaries. Therefore, another limitation is the lack of control of other determinants of anthropometric conformation. Further experimental researches are needed to investigate on long-time observation of the phenomena, also checking whether this equivalence is maintained over time or if there are differences in performance related to other training methodologies.

\section{Conflicts of interest}

The Authors certify that there is no conflict of interest with any financial organization regarding the material discussed in the manuscript.

\section{References}

1. Joondeph SA, Joondeph BC. Retinal Detachment due to CrossFit Training Injury. Case Rep Ophthalmol Med. 2013;2013:189837.

2. Weiss T, Kreitinger J, Wilde H, Wiora C, Steege M, Dalleck L, Janot, J. Effect of Functional Resistance Training on Muscular Fitness Outcomes in Young Adults. J Exerc Sci Fit. 2010; 8(2):113-122.

3. Brill P. Exercise your independence: Functional fitness for older adults. Human Kinetics 2004.

4. Boyle M. New Functional Training for Sports. Human Kinetics. 2016.

5. Tafuri S, Notarnicola A, Monno A, Ferretti F, Moretti B. CrossFit athletes exhibit high symmetry of fundamental movement patterns. A cross-sectional study. Muscles Ligaments Tendons J. 2016 May 19;6(1):157-160.

6. Buchner DM, Larson EB, Wagner EH, Koepsell TD, de Lateur BJ. Evidence for a non-linear relationship between leg strength and gait speed. Age and Ageing. 1996;25(5):386391.
7. Kavanagh JJ, Menz HB. Accelerometry: a technique for quantifying movement patterns during walking. Gait \& Posture. 2008;28(1):1-15.

8. Neves LM, Fortaleza AC, Rossi FE, Diniz TA, Codogno JS, Gobbo $L A$, et al. Functional training reduces body fat and improves functional fitness and cholesterol levels in postmenopausal women: a randomized clinical trial. J Sports Med Phys Fitness. 2017 Apr;57(4):448-456.

9. Heinrich KM, Becker C, Carlisle T, Gilmore K, Hauser J, Frye $J$, Harms CA. High-intensity functional training improves functional movement and body composition among cancer survivors: a pilot study. Eur J Cancer Care (Engl). 2015 Nov;24 (6):812-817.

10. Poston WS, Haddock CK, Heinrich KM, Jahnke SA, Jitnarin N, Batchelor DB. Is High-Intensity Functional Training (HIFT) /CrossFit Safe for Military Fitness Training? Mil Med. 2016 Jul;181(7):627-637.

11. Glassman G. Understanding CrossFit. The CrossFit Journal. 2007;56:1.

12. Poston WS, Haddock CK, Heinrich KM, Jahnke SA, Jitnarin N, Batchelor DB. Is High-Intensity Functional Training (HIFT)/ CrossFit Safe for Military Fitness Training? Mil Med. 2016 Jul;181(7):627-637.

13. Butcher SJ, Neyedly TJ, Horvey KJ, Benko CR. Do physiological measures predict selected CrossFit( $(B)$ benchmark performance? Open Access J Sports Med. 2015;6:241-247.

14. Choi EJ, So WY, Jeong TT. Effects of the CrossFit Exercise Data Analysis on Body Composition and Blood Profiles. Iran J Public Health. 2017 Sep;46(9):1292-1294.

15. Eather N, Morgan PJ, Lubans DR. Improving health-related fitness in adolescents: the CrossFit Teens ${ }^{\mathrm{TM}}$ randomised con- $^{-}$ trolled trial. J Sports Sci. 2016;34(3):209-223.

16. Tinto $A$, Campanella $M$, Fasano $M$. Core strengthening and synchronized swimming: TRX® suspension training in young female athletes. J Sports Med Phys Fitness. 2017 Jun;57(6): 744-751.

17. Padulo J, Oliva F, Frizziero A, Maffulli N. Muscles, Ligaments and Tendons Journal - Basic principles and recommendations in clinical and field science research: 2016 update. MLTJ. 2016;6(1):1-5.

18. https://crossfit.com/

19. https://www.trxtraining.com/\#

20. WHO. Physical status: the use and interpretation of anthropometry. Report of a WHO Expert Committee. WHO Technical Report Series 854. Geneva: World Health Organization, 1995.

21. Ashwell M, Mayhew L, Richardson J, Rickayzen B. Waist-toheight ratio is more predictive of years of life lost than body mass index. PLoS ONE. 2014;9(9).

22. Yoo EG. Waist-to-height ratio as a screening tool for obesity and cardiometabolic risk. Korean J Pediatr. 2016;59(11):425431.

23. Jackson AS, Pollock ML. Generalized equations for predicting body density of men. Br J Nutr. 1978; 40(3):497.

24. Balke B, Ware RW. An experimental study of physical fitness of Air Force personnel. United States Armed Forces Medical Journal. 1959;10:675-688.

25. The Physical Fitness Specialist Certification Manual, The Cooper Institute for Aerobics Research, Dallas TX, revised 1997, printed in Advance Fitness Assessment \& Exercise Prescription, 3rd Edition, Vivian H. Heyward. 1998;48.

26. Sargent DA. The Physical Test of a Man. Am Phys Edu Rev. 1921;26:188-194.

27. Davis B. Physical Education and the study of sport. 4th ed. London: Harcourt Publishers. 2000;123.

28. Dodd DJ, Alvar BA. Analysis of acute explosive training modalities to improve lower-body power in baseball players. J Strength Cond Res. 2007;21(4):1177-1182. 
29. Farrar RE, Mayhew JL, Koch AJ. Oxygen cost of kettlebell swings. J Strength Cond Res. 2010;24(4):1034-1036.

30. Bellar D, Judge LW. Modeling and relationship of respiratory exchange ratio to athletic performance. J Strength Cond Res. 2012;26(9):2484-2489.

31. Garhammer J. Power production by Olympic weightlifters. Medicine and Science in Sports and Exercise. 1980;12(1):54-60.

32. Garhammer JA. Comparison of Maximal Power Outputs Between Elite Male and Female Weightlifters in Competition. In J Sport Biomech. 1991;7:3-1.

33. Manocchia P, Spierer DK, Lufkin AKS, Minichiello J, Castro J.
Transference Of Kettlebell Training To Strength, Power, And Endurance. 2013;27(2):477-484.

34. Pollock ML, Gaesser GA, Butcher JD, Després JP, Dishman RK, Franklin BA, Garber CE. American College of Sports Medicine Position Stand. The recommended quantity and quality of exercise for developing and maintaining cardiorespiratory and muscular fitness, and flexibility in healthy adults. Medicine and Science in Sports and Exercise. 1998;30 (6):975-991.

35. Stone MH. Muscle conditioning and muscle injuries. MedSci Sports Exerc. 1990;22(4):457-462. 